\title{
Le financement de la transmission/reprise des PME : Un tour d'horizon ${ }^{1}$
}

\author{
Louise Cadieux, DBA et Pierre Gratton, Doctorant au DBA \\ Université du Québec à Trois-Rivières
}

\section{INTRODUCTION}

Au Québec, on estime que 30,4 \% des propriétaires-dirigeants actifs en 2008 quitteront leur entreprise pour 2018, dont 13,3\% avant 2013.

La transmission/reprise des PME est une problématique de plus en plus actuelle. Cela s'explique par une pyramide des âges vieillissante des propriétaires dirigeants en poste susceptibles de passer la direction et la propriété de leur PME à une nouvelle génération d'entrepreneurs prêts à relever des défis différents de ceux d'une création pure (ex nihilo). Par exemple, au Québec, on estime que 30,4\% des propriétaires-dirigeants actifs en 2008 quitteront leur entreprise pour 2018, dont $13,3 \%$ avant 2013. Alors que $19,4 \%$ des nouveaux entrepreneurs envisagent une carrière entrepreneuriale par le biais d'une reprise d'entreprise familiale, interne ou externe ${ }^{2}$. Dans une perspective économique, l'enjeu est capital car la réussite des projets de transmission ou de reprise des PME présentent des avantages importants allant au-delà du maintien des emplois et de la production des pays concernés par le phénomène. Malgré la difficulté perçue de la part du cédant et du repreneur, au plan de la transmission/reprise des $\mathrm{PME}^{3}$, plusieurs programmes d'aide financière sont offerts. Toutefois, à ce jour, aucun document ne brosse un portrait exhaustif de l'offre de financement en transmission/reprise, notamment pour les PME québécoises. Cet article a donc pour principal objectif de faire un tour d'horizon des programmes d'aide financière en transmission/reprise et, dans la foulée, mettre en exergue leurs principales forces et faiblesses de sorte à faire une meilleure lecture de l'offre globale existante à cet effet.

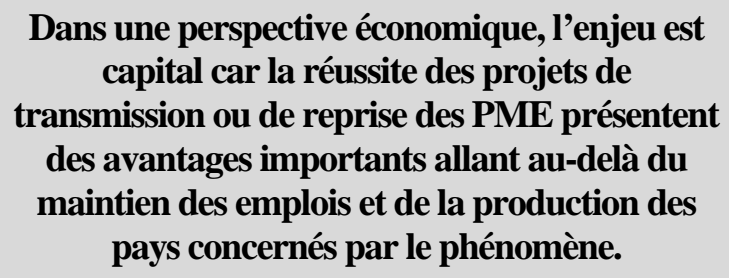

\section{LE FINANCEMENT DE LA TRANSMISSION/REPRISE : UNE MISE EN CONTEXTE}

La problématique financière de la transmission/reprise revêt diverses facettes tant pour le cédant que pour le repreneur. La difficulté à déterminer la valeur de l'entreprise, les coûts afférents au transfert de propriété, les coûts cachés du transfert de pouvoir, les coûts d'achat eux-mêmes ainsi que les besoins financiers nécessaires à la relance de l'entreprise acquise sont quelques exemples. Pour le repreneur, il ne s'agit donc pas de prévoir un simple transfert de fonds lors de l'achat de l'entreprise ciblée, mais bien de planifier en profondeur ses besoins en financement pour plusieurs années. En général, les projets de transmission/reprise de moindre envergure se financent directement par les capitaux propres, soit un montage financier classique comprenant entre $20 \%$ et $30 \%$ de mise de fonds, des emprunts bancaires couvrant entre $40 \%$ et $50 \%$ des besoins et un crédit accordé par le cédant pour financer les $20 \%$ à $30 \%$ restants. Dans le cas d'une transmission/reprise plus importante, le repreneur a recours aux capitaux empruntés tels que les différents types de financement subordonné ainsi que le financement par effet de levier (LBO). Au Québec, plusieurs institutions financières, notamment la Caisse de dépôt et placement du Québec et le Fonds de solidarité FTQ, facilitent ce genre de financement ${ }^{4}$. En 2005, les résultats d'une étude sur le sujet 
montraient qu'au Québec, tant les cédants que les repreneurs de PME de moyenne et grande taille avaient accès à une offre de financement variée et adaptée à leurs besoins. Davantage l'affaire des institutions bancaires classiques, les programmes de financement visant les très petites entreprises (TPE) étaient cependant moins nombreux puisque délaissés par les intervenants financiers spécialisés (fonds d'investissement), ces derniers étant plutôt

\section{LA DÉMARCHE MÉTHODOLOGIQUE}

Pour connaître les programmes d'aide financière consacrés à la transmission/reprise des PME québécoises, nous avons opté pour différentes méthodes de travail. D'abord, après avoir fait un retour sur les ressources Internet déjà identifiées par notre groupe de recherche en 2008, nous avons, dans le cadre de cette étude faite en novembre 2010, revisité tous les sites Internet des organismes ou organisations s'intéressant à la relève d'entreprise. Lorsque les informations

\section{LES RÉSULTATS}

Dans le cadre de cette recherche, nous avons répertorié trois principales catégories de ressources financières disponibles pour la transmission/reprise des PME. Il s'agit des organismes gouvernementaux et de première ligne, des institutions financières et des fonds d'investissement. Nous les présenterons donc, dans cet ordre, dans les pages qui suivent.

\subsection{Les organismes gouvernementaux et de première ligne}

Soucieux du maintien du dynamisme économique régional et/ou national, les gouvernements canadiens et québécois ont constitué différents organismes et favorisé une structure plus locale afin de faciliter l'aide accordée dans les régions.

Soucieux du maintien du dynamisme économique régional et/ou national, les gouvernements canadiens et québécois ont constitué différents organismes et favorisé une structure plus locale afin de faciliter l'aide accordée dans les régions. Le maintien ou la création des intéressés par les financements via des participations dans les fonds propres d'entreprises de plus grande taille ${ }^{5}$. Malgré ce que nous pouvons retenir des travaux antérieurs, force est de constater que la question demeure entière : la problématique de la transmission/reprise de nos PME étant de plus en plus préoccupante, qu'en est-il au juste de l'offre de financement en la matière en 2010 ?

étaient difficiles à interpréter, nous avons contacté par téléphone des personnes ressources, s'assurant ainsi d'un inventaire le plus représentatif de la réalité. Enfin, toujours dans la même logique, nous avons aussi rencontré des personnes ressources œuvrant dans différents milieux, qui, pour leur part, ont enrichi notre compréhension du portefeuille d'aide financière consacré à la transmission/reprise des PME.

emplois, l'exode des jeunes, la conservation des compétences clés sont quelques exemples des préoccupations soulevées par ceux-ci. Parmi les organismes gouvernementaux et de première ligne offrant de l'aide financière à la transmission/reprise, nous en retenons quatre qui sont la Financière Agricole; Financement agricole Canada; les réseaux des Société d'aide au développement des collectivités (SADC) et les centres locaux de développement du Québec (CLD). Comme le résume le tableau 1, pour les deux premiers organismes, il s'agit de programmes d'aide financière s'adressant aux entreprises du secteur de l'agriculture. Dans leur cas, en amont du financement, existent les réseaux Agriconseils. Hormis leur première mission qui est d'accompagner les agriculteurs actuels ou en devenir, Agriconseils offre de l'aide financière sous forme de remboursement d'une partie des frais de consultation, notamment pour le montage financier de la transmission/reprise d'entreprises agricoles. Selon les programmes de la Financière Agricole et Financement agricole Canada, l'aide peut prendre la forme de subventions, de prêts, de garantie de prêts ou 
d'émissions de certificats de prêts vers une institution financière. L'aide financière est accordée sous réserve de certaines conditions dont l'âge et la formation du repreneur et/ou la présentation d'un plan d'affaires bien articulé par exemple. Les programmes retenus pour les transmissions/reprises du secteur de l'agriculture sont distincts mais aussi complémentaires dans la mesure où certains proposent de l'aide au cédant, d'autres au repreneur tant en matière de financement que d'accompagnement tout au long des différents cycles de vie de l'entreprise.

Pour le financement offert par le réseau des SADC, les programmes se distinguent par leur raison d'être : le maintien de la vitalité des tissus économiques dans les régions considérées plus à risque.

Pour le financement offert par le réseau des SADC, les programmes se distinguent par leur raison d'être : le maintien de la vitalité des tissus économiques dans les régions considérées plus à risque, notamment au chapitre de l'entrepreneuriat et de la main-d'œuvre. Les programmes excluent donc les grands centres comme Montréal, Québec, Laval et Gatineau et s'adressent principalement au nouvel entrepreneur (donc le repreneur, dans la majorité des cas), incluant une restriction d'âge, dans certains cas. Dans l'ensemble du réseau des SADC, l'aide financière est offerte sous forme de certificats de prêts, de prêts conventionnels, de partage de capital-actions ou de congé d'intérêts et de remboursement pour les cinq premières années. Les programmes visent les projets de démarrage, d'acquisition ou d'expansion et sont souvent complémentaires à des institutions financières régionales. Parmi les conditions exigées, nous retenons l'obligation pour le bénéficiaire d'œuvrer dans la région prêteuse, la logique du dynamisme économique régional et national étant présente en arrière-plan, cela plus précisément dans certaines régions. Par exemple, les SADC de Témiscouata et de la Côte-Nord exigent que le siège social de l'entreprise financée soit dans la région, alors que pour d'autres régions, cela ne l'est pas. Notons aussi que, dans la majorité des cas, les programmes incluent des services d'accompagnement per- mettant au nouvel entrepreneur (donc au repreneur) de bien cheminer dans son projet de vie professionnelle.

Parmi les organismes de première ligne, nous avons aussi retenu les programmes d'aide financière offerts dans le réseau des CLD. Par le biais de l'association des CLD (ACLD), deux ont attiré notre attention: les Fonds locaux d'investissement (FLI), et les Fonds jeunes promoteurs. Toutefois, certains CLD offrent d'autres programmes spécifiques aux besoins de leur région. Selon les programmes, il peut s'agir de subventions ou d'émission de certificats de prêts. Dans leur cas, l'analyse de l'offre de financement est plus complexe puisque les CLD mettent en place des programmes personnalisés, selon les particularités et les besoins de leurs régions respectives. Notons enfin que l'aide financière peut être prodiguée au cas par cas dans plusieurs régions et porter plusieurs étiquettes. Par exemple, au CLD de Mékinac, existe le programme FLI, volet relève offert sous forme de subventions et le Fonds d'Aide à la Relève, sous forme de prêts. Alors qu'au CLD $\mathrm{du}$ Haut-St-Maurice, il y a le Fonds Relève Affaires offert sous forme de subvention et le Fonds d'Aide à la Relève, sous forme de prêts. Pour résumer, les programmes offerts par les CLD permettent au repreneur d'avoir une aide financière pouvant varier selon les régions tant au chapitre des montants accordés qu'à celui des conditions préalables, comme son expérience professionnelle, son âge, le secteur d'activité ou les orientations des politiques d'investissement régionales. Enfin, les CLD offrent aussi des services d'accompagnement pouvant prendre la forme d'aide à l'élaboration du plan d'affaire, la recherche de financement ou la gestion de l'entreprise et la formation des nouveaux entrepreneurs (incluant les repreneurs).

\section{Les programmes offerts par les CLD} permettent au repreneur d'avoir une aide financière pouvant varier selon les régions tant au chapitre des montants accordés qu'à celui des conditions préalables, comme son expérience professionnelle, son âge, le secteur d'activité ou les orientations des politiques d'investissement régionales. 


\section{Tableau 1 : Aide financière provenant des organismes publics et de première ligne}

\begin{tabular}{|c|c|c|c|c|}
\hline \multicolumn{5}{|c|}{ Organismes publics } \\
\hline $\begin{array}{l}\text { Secteurs } \\
\text { couverts }\end{array}$ & Bénéficiaires & $\begin{array}{l}\text { Types de } \\
\text { transmissions } \\
\text { /reprises }\end{array}$ & $\begin{array}{l}\text { Aide } \\
\text { financière }\end{array}$ & Particularités des programmes d'aide financière \\
\hline \multicolumn{5}{|c|}{ Financière Agricole; Financement Agricole Canada } \\
\hline $\begin{array}{l}\text { Agri- } \\
\text { culture }\end{array}$ & $\begin{array}{l}\text { Cédants } \\
\text { Repreneurs }\end{array}$ & $\begin{array}{l}\text { Familiale } \\
\text { Interne } \\
\text { Externe }\end{array}$ & $\begin{array}{l}\text { Jusqu'à } \\
5000000 \$\end{array}$ & $\begin{array}{l}\text { - Objectif : sauvegarder les entreprises œuvrant dans le } \\
\text { secteur de l'agriculture } \\
\text { - Aides financières sous forme de subventions, de prêts, de } \\
\text { garanties de prêts, de certificats de prêts ou de taux } \\
\text { d'intérêts plafonnés (rabais) } \\
\text { - Mises de fonds minimum exigées pour le cédant ou le } \\
\text { repreneur } \\
\text { - Programmes, selon l'avancement du projet de } \\
\text { transmission/reprise } \\
\text { - Conditions de formation du repreneur et/ou du dépôt d'un } \\
\text { plan d'affaire } \\
\text { - Restriction d'âge minimum du repreneur (+18 ans) } \\
\text { - Restriction de citoyenneté canadienne ou résident } \\
\text { permanent } \\
\text { - Montages financiers accompagnés ou supervisés par } \\
\text { Agriconseils }\end{array}$ \\
\hline \multicolumn{5}{|c|}{ Réseaux SADC } \\
\hline $\begin{array}{l}\text { Selon les } \\
\text { régions }\end{array}$ & Repreneurs & $\begin{array}{l}\text { Selon les } \\
\text { programmes } \\
\text { des SADC } \\
\text { régionales }\end{array}$ & $\begin{array}{l}\text { Jusqu'à } \\
500000 \$\end{array}$ & $\begin{array}{l}\text { - Objectif : sauvegarder le dynamisme économique des } \\
\text { régions } \\
\text { - Exclut Montréal, Québec, Laval et Gatineau } \\
\text { - Aide financière sous forme de prêts, d'émission de } \\
\text { certificats de prêts, de participation au capital-actions, de } \\
\text { congé d'intérêt et de remboursement pour } 5 \text { ans } \\
\text { - Mise de fonds minimale exigée } \\
\text { - Restrictions de formation ou d'expérience du repreneur } \\
\text { - Certaines restrictions d'âge (18 à } 35 \text { ans) peuvent } \\
\text { s'appliquer, selon les programmes et les régions } \\
\text { - Obligation d'œuvrer dans la région prêteuse } \\
\text { - Obligation de s'inscrire dans les orientations de } \\
\text { développement régionales } \\
\text { - Obligation de s'associer à d'autres partenaires } \\
\text { - Services d'accompagnement }\end{array}$ \\
\hline \multicolumn{5}{|c|}{ Réseaux CLD, CDÉ, IDÉ .... } \\
\hline $\begin{array}{l}\text { Selon les } \\
\text { régions }\end{array}$ & Repreneurs & $\begin{array}{l}\text { Selon les } \\
\text { programmes } \\
\text { offerts dans } \\
\text { les régions }\end{array}$ & $\begin{array}{l}\text { Jusqu'à } \\
150000 \$\end{array}$ & $\begin{array}{l}\text { - Objectif : sauvegarder le dynamisme entrepreneurial } \\
\text { québécois } \\
\text { - Aides financières sous forme de subventions ou certificats } \\
\text { de prêts } \\
\text { - À la pièce, selon les organismes locaux ou régionaux } \\
\text { - Certaines restrictions d'âge ( } 18 \text { à } 35 \text { ans) peuvent } \\
\text { s'appliquer } \\
\text { - Obligation de s'inscrire dans les orientations politiques } \\
\text { d'investissement du CLD } \\
\text { - Mises de fonds exigées } \\
\text { - Minimum de } 5 \text { employés } \\
\text { - Services d'accompagnement et de formation }\end{array}$ \\
\hline
\end{tabular}




\subsection{Les institutions financières}

Les institutions financières recherchent plutôt des projets dans lesquels elles injectent des mises de fonds en lien avec la capacité financière du cédant ou du repreneur, leur tolérance au risque étant plus faible que pour les organismes de fonds d'investissement.

Pour les institutions financières, nous en avons retenu neuf. Tel que présenté au tableau 2, il s'agit de la Banque Nationale, du Mouvement Desjardins, de la Banque Scotia, de la Banque de Montréal, de la Banque CIBC, de la Banque Royale, de la Toronto Dominion Canada Trust, de La Banque Laurentienne et de la Banque HSBC. Les institutions financières ont, depuis toujours, des programmes dédiés à la transmission/reprise d'entreprise et cela même si la majorité ne le mettent pas en évidence sur leurs sites Internet. Leur proximité avec leur clientèle d'affaires explique cette situation, surtout lorsqu'il s'agit d'accompagner des PME fructueuses avec lesquelles elles entretiennent des liens de confiance solides depuis plusieurs années. Pour la transmission/reprise, les institutions financières recherchent plutôt des projets dans lesquels elles injectent des mises de fonds en lien avec la capacité financière du cédant ou du repreneur, leur tolérance au risque étant plus faible que pour les organismes de fonds d'investissement.

Les institutions financières sont, par ailleurs, une ressource facile d'accès pour le cédant et le repreneur favorisant des montages financiers en partenariat avec d'autres organismes, lorsque le risque est plus élevé. Enfin, nous retenons que les programmes sont offerts non seulement aux cédants et aux repreneurs de tous types (familiaux, internes, externes), mais sont aussi personnalisés et incluent des services d'accompagnement et de conseils.

\section{Tableau 2 : Aide financière provenant des institutions financières}

\begin{tabular}{|c|c|c|c|c|}
\hline \multicolumn{5}{|c|}{ Institutions financières } \\
\hline $\begin{array}{l}\text { Secteurs } \\
\text { couverts }\end{array}$ & Bénéficiaires & $\begin{array}{l}\text { Types de } \\
\text { transmissions } \\
\text { /reprises }\end{array}$ & $\begin{array}{l}\text { Aide } \\
\text { financière }\end{array}$ & Particularités des programmes d'aide financière \\
\hline \multicolumn{5}{|c|}{$\begin{array}{l}\text { Banque Nationale; Mouvement Desjardins; Banque Scotia; Banque de Montréal; Banque CIBC; Banque Royale; TD } \\
\text { Canada Trust; Banque Laurentienne; Banque HSBC }\end{array}$} \\
\hline $\begin{array}{l}\text { Aucune } \\
\text { spécification } \\
\text { en ce sens }\end{array}$ & $\begin{array}{l}\text { Cédants } \\
\text { Repreneurs }\end{array}$ & $\begin{array}{l}\text { Familiale } \\
\text { Interne } \\
\text { Externe }\end{array}$ & Cas par cas & $\begin{array}{l}\text { - Objectif : offrir un portefeuille de produits diversifié à } \\
\text { la clientèle de sorte à assurer leur fidélisation } \\
\text { - Aide financière sous forme de prêts bancaires, } \\
\text { principalement } \\
\text { - Capacité financière du cédant ou du repreneur prise en } \\
\text { compte } \\
\text { - Existence de certains programmes spécifiques depuis } \\
\text { quelques années (Banque Nationale, Desjardins, etc.) } \\
\text { - Partenariat et complémentarité avec d'autres } \\
\text { programmes (capitaux de risque, SADC, Agriculture, } \\
\text { etc.) } \\
\text { - Services d'accompagnement (suivis, évaluation, } \\
\text { planification relève, etc.) }\end{array}$ \\
\hline
\end{tabular}




\subsection{Les fonds d'investissement}

\section{La majorité des programmes provenant des fonds d'investissement s'adressent à des entreprises considérées saines financièrement, ayant des bonnes pratiques de gestion et des objectifs de croissance ou de diversification.}

Les organismes de fonds d'investissement offrent des programmes d'aide financière à la transmission/reprise des PME principalement pour assurer le maintien du dynamisme économique régional et national par des capitaux québécois. Comme le résume le tableau 3, pour cette catégorie, nous avons retenu huit organisations: Desjardins Capital de risques (DCR), les Fonds de Solidarité FTQ, Fondaction CSN, Investissement Québec (IQ), la Société Générale de Financement (SGF) ${ }^{6}$, la Société d'Investis- sement Jeunesse (SIJ), la Caisse de Dépôt et de Placement du Québec (CDP) et la Banque de développement du Canada (BDC). La majorité des programmes provenant des fonds d'investissement s'adressent à des entreprises considérées saines financièrement, ayant des bonnes pratiques de gestion et des objectifs de croissance ou de diversification. Dans leur cas, l'aide financière peut prendre la forme de garanties de prêts, de prêts à termes garantis, de participation au capital-actions, de débentures, etc. Depuis plusieurs années, chaque programme offert par les fonds d'investissement a une mission claire reconnue, une clientèle spécifique souvent aussi clairement déterminée de même que des conditions pouvant, dans une perspective globale, rejoindre la majorité des cédants ou des repreneurs à la recherche de financement pour leur projet de transmission ou de reprise.

Tableau 3 : Aide financière provenant des fonds d'investissement

\begin{tabular}{|c|c|c|c|c|}
\hline \multicolumn{5}{|c|}{ Fonds d'investissement } \\
\hline $\begin{array}{l}\text { Secteurs } \\
\text { couverts }\end{array}$ & Bénéficiaires & $\begin{array}{l}\text { Types de } \\
\text { transmissions } \\
\text { /reprises }\end{array}$ & $\begin{array}{l}\text { Aide } \\
\text { financière }\end{array}$ & Particularités des programmes d'aide financière \\
\hline \multicolumn{5}{|c|}{$\begin{array}{l}\text { Desjardins Capital de risques; Fonds de Solidarité FTQ; Fondaction CSN; Investissement Québec; Société Générale de } \\
\text { Financement; Société d'Investissement Jeunesse; Caisse de Dépôt et de Placement; Banque de développement du } \\
\text { Canada }\end{array}$} \\
\hline $\begin{array}{l}\text { Tous } \\
\text { types }\end{array}$ & $\begin{array}{l}\text { Cédants } \\
\text { Repreneurs }\end{array}$ & $\begin{array}{l}\text { Familiale } \\
\text { Interne } \\
\text { Externe }\end{array}$ & $\begin{array}{l}\text { Maximum de } \\
25000000 \$\end{array}$ & $\begin{array}{l}\text { - Objectif : maintien des capitaux régionaux et nationaux } \\
\text { - Aide diversifiée pouvant prendre la forme de garanties } \\
\text { de prêts, de prêts à termes garantis, de participation au } \\
\text { capital-actions, de débentures, etc. } \\
\text { - S'adressent aux entreprises ayant une structure financière } \\
\text { et de gestion saine, dans certains cas } \\
\text { - Plans de croissance ou de diversification souvent exigés } \\
\text { - Conditions d'investissement minimum de la part du } \\
\text { bénéficiaire } \\
\text { - Conditions de sorties pour l'organisme de fonds } \\
\text { d'investissement (temps, retour sur investissement, etc.) } \\
\text { - S'adressent aux entreprises ayant un chiffre d'affaires ou } \\
\text { une valeur marchande minimum et/ou maximum } \\
\text { - Implique des négociations entre les parties et nécessite la } \\
\text { préparation et la signature de plusieurs documents } \\
\text { juridiques } \\
\text { - Incluent la présence des fonds d'investissement dans la } \\
\text { gouvernance de l'entreprise (conseil d'administration; } \\
\text { comité de gestion, etc.) } \\
\text { - Programmes complémentaires aux institutions } \\
\text { financières } \\
\text { - Le critère d'âge est absent, sauf dans le cas d'un } \\
\text { programme spécifique qui s'adresse aux jeunes (SIJ) (18- } \\
35 \text { ans) } \\
\text { - Services de consultation, de formation et } \\
\text { d'accompagnement }\end{array}$ \\
\hline
\end{tabular}




\section{LES FORCES ET LES FAIBLESSES DES PROGRAMMES D’AIDE FINANCIÈRE RETENUS}

\section{Les programmes offerts dans le secteur de l'agriculture se démarquent, car il y a une prise de conscience de nos gouvernements et du milieu de la fragilité de ce secteur.}

Parmi l'ensemble des offres de financement dédiées à la transmission/reprise que nous avons retenues dans le cadre de cette étude, certaines forces et faiblesses présentées au tableau 4 méritent d'être soulignées. D'abord, les programmes offerts dans le secteur de l'agriculture se démarquent ${ }^{7}$, car il y a une prise de conscience de nos gouvernements et du milieu de la fragilité de ce secteur depuis les dernières années, une prise en charge et des actions posées qui ont suivi. Selon le programme, l'aide financière est disponible pour le cédant et pour le repreneur. L'offre globale assure le développement des compétences du repreneur par le biais de la formation, de l'expérience et/ou de l'élaboration d'un plan d'affaires structuré. Des services conseils et d'accompagnement subventionnés et ayant fait leurs preuves au fil des ans sont également proposés aux bénéficiaires en amont du processus de montage financier de la transmission/reprise. Notons, au passage, que parmi les forces des programmes offerts dans le secteur de l'agriculture, le MAPAQ a, depuis plusieurs années, une politique d'exemption en gains en capital pour le transfert d'un bien agricole à la famille, facilitant ainsi le processus de transfert des fermes familiales.

$\mathrm{Au}$ reste, bien que l'information sur les programmes d'aide de financement pour la transmission/reprise soit facile d'accès sur les sites Internet des organismes, il semble qu'ils manquent de visibilité pour certains bénéficiaires. Par exemple, lors d'une activité organisée par la MCR de Maskinongé, une repreneuse familiale, accompagnée d'une conseillère du centre régional d'établissement en agriculture du Québec (CREA), avouait avoir trouvé l'accompagnement dont elle avait besoin par accident alors que cela faisait plusieurs années qu'elle cherchait de l'aide dans son processus de relève déjà bien amorcé. Cela dit, nous comprenons que ce ne sont pas tous les cédants ni les repreneurs qui ont le réflexe de chercher l'information dont ils ont besoin sur les sites Internet des organismes gouvernementaux. Au même titre que ce ne sont pas tous les cédants ou les repreneurs qui ont pour habitude de fréquenter les activités organisées par les CREA régionaux où l'information sur lesdits programmes circule bien. Enfin, la principale faiblesse notée pour les programmes d'aide à la relève agricole découle des outils utilisés lesquels demeurent souvent centrés autour des transmissions/reprises familiales alors que l'offre s'adresse à tous les types de transmission/reprise.

En ce qui concerne les programmes offerts par les organismes de première ligne, l'aide financière pour la transmission/reprise est incluse dans ceux s'adressant aux personnes désireuses de "partir en affaires », dont les créations de toutes pièces (ex nihilo), notant ainsi une cohérence avec les objectifs desdits programmes : dynamiser le tissu entrepreneurial régional et national. Cependant, il est moins évident pour les cédants et les repreneurs à la recherche d'aide dans leurs projets respectifs.

Les principales forces retenues des programmes provenant des organismes de première ligne résident dans leur souplesse, dans les mises de fonds exigées souvent minimes, dans l'obligation pour le nouvel entrepreneur de présenter un plan d'affaires et dans les services d'accompagnement et de formation offerts. Hormis un éventail de programmes d'aide financière intéressant, plusieurs faiblesses ont été notées. Il est aussi possible de retenir, dans le cas du réseau des SADC, l'exclusion des grands centres, alors qu'il y existe plusieurs entreprises de toutes tailles ou de tous types à transmettre ou à reprendre. La deuxième faiblesse est la centralisation des programmes offerts par les SADC et les CLD autour des orientations politiques d'investissement régionales dans la mesure où elles peuvent changer selon les personnes ressources régionales en poste. Ces faiblesses rendent confuses et diffuses les offres et les conditions d'admission pour les bénéficiaires qui, souvent, ne savent pas comment faire pour trouver du financement ou ne comprennent pas à qui s'adresser pour avoir l'aide souhaitée. 


\section{Tableau 4 : Les forces et les faiblesses des programmes d'aide financière}

\begin{tabular}{|c|c|c|}
\hline & Forces & Faiblesses \\
\hline 茪 & $\begin{array}{l}\text { - Bénéficiaires cédants et repreneurs } \\
\text { - Aucune restriction d'âge maximum } \\
\text { - Formation du repreneur ou plan d'affaire exigé } \\
\text { - Aide financière sous forme de subventions } \\
\text { - Mises de fonds minimes } \\
\text { - Programmes d'accompagnement subventionnés en } \\
\text { amont du processus de relève } \\
\text { - Présence des CREA } \\
\text { - Règles sur l'imposition d'un transfert ou une vente } \\
\text { agricole (familiale) } \\
\text { - Engagement clair et concertation de plusieurs } \\
\text { parties prenantes (gouvernements, UPA, etc.) }\end{array}$ & $\begin{array}{l}\text { - Centralisation autour des transmissions familiales } \\
\text { (historique des programmes) } \\
\text { - L'offre peut être difficile à trouver pour les éventuels } \\
\text { bénéficiaires qui ne sont pas familiers avec les sites } \\
\text { Internet }\end{array}$ \\
\hline 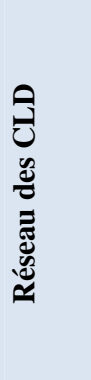 & $\begin{array}{l}\text { - Proximité } \\
\text { - Portefeuille adapté d'aide financière, } \\
\text { complémentaire aux fonds d'investissement et aux } \\
\text { institutions financières } \\
\text { - Expérience du repreneur ou plan d'affaire exigé } \\
\text { - Mises de fonds parfois minimes } \\
\text { - Souplesse des conditions } \\
\text { - Accompagnement }\end{array}$ & $\begin{array}{l}\text { - Centralisation autour des orientations politiques } \\
\text { régionales } \\
\text { - Offre diffuse pouvant être difficile à trouver ou à } \\
\text { comprendre pour les éventuels bénéficiaires Logique } \\
\text { - entrepreneuriale (démarrage d'entreprise) } \\
\text { - Bénéficiaires repreneurs seulement } \\
\text { - Certaines restrictions d'âges (18 à } 35 \text { ans) } \\
\text { - Services d'accompagnement } \\
\text { - Manque d'homogénéité dans la présentation de } \\
\text { programmes qui sont souvent les mêmes }\end{array}$ \\
\hline 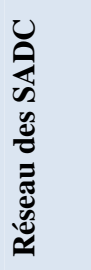 & $\begin{array}{l}\text { - Distinctions régionales } \\
\text { - Bon portefeuille d'aide financière, complémentaire } \\
\text { aux fonds d'investissement et aux institutions } \\
\text { financières }\end{array}$ & $\begin{array}{l}\text { - Exclusion des grands centres } \\
\text { - Bénéficiaires repreneurs seulement } \\
\text { - Certaines restrictions d'âges (18 à } 35 \text { ans) } \\
\text { - Manque d'homogénéité dans la présentation d'une une } \\
\text { offre qui est souvent la même } \\
\text { - Offre diffuse pouvant être difficile à trouver ou à } \\
\text { comprendre pour les éventuels bénéficiaires }\end{array}$ \\
\hline 总总 & $\begin{array}{l}\text { - Proximité de la clientèle } \\
\text { - Service personnalisé (cas par cas) } \\
\text { - Lien de confiance } \\
\text { - Montage financier impliquant plusieurs } \\
\text { partenaires : distribution du risque }\end{array}$ & $\begin{array}{l}\text { - Clientèle ciblée (entreprises saines, etc.) liée au risque } \\
\text { d'affaires des institutions financières souvent frileuses } \\
\text { - Services conseils et d'accompagnement souvent très } \\
\text { centralisés autour du transfert du capital-actions, des } \\
\text { actifs ou du patrimoine }\end{array}$ \\
\hline 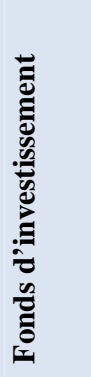 & $\begin{array}{l}\text { - Expérience } \\
\text { - Réputation } \\
\text { - Portefeuille d'aide financière } \\
\text { - Accessibilité pour tous les secteurs d'activité } \\
\text { - Bénéficiaires cédants et repreneurs } \\
\text { - Partenariats avec les institutions financières } \\
\text { - Inclusion des biens intangibles } \\
\text { - Complémentarité des programmes } \\
\text { - Accompagnement }\end{array}$ & $\begin{array}{l}\text { - Conditions d'admission (taille, croissance, santé } \\
\text { financière, etc.) } \\
\text { - Conditions imposées par les gestionnaires de fonds } \\
\text { (droits de gestion, etc.) } \\
\text { - Clientèle ciblée (entreprises saines, etc.) liée au risque } \\
\text { d'affaires des fonds d'investissement } \\
\text { - Difficulté pour les bénéficiaires de comprendre les } \\
\text { particularités de chacun des programmes offerts par les } \\
\text { différents fonds d'investissement (critères disparates, } \\
\text { etc.) }\end{array}$ \\
\hline
\end{tabular}




\section{Comme dans le cas des organismes publics, les programmes d'aide à la transmission/reprise des institutions financières incluent des services conseils et d'accompagnement, toutefois souvent très centralisés autour du transfert du capital- actions, des actifs ou du patrimoine.}

Quant aux institutions financières, il semble que leurs principales forces découlent de leur proximité avec la clientèle. Depuis qu'elles existent, elles offrent un service personnalisé et font $\mathrm{du}$ cas par cas. C'est une raison qui expliquerait la nature peu concluante de l'information sur la transmission/reprise disponible sur leurs sites Internet pour quiconque ne fait pas affaires avec elles. Comme dans le cas des organismes publics, les programmes d'aide à la transmission/reprise des institutions financières incluent des services conseils et d'accompagnement, toutefois souvent très centralisés autour du transfert du capital-actions, des actifs ou du patrimoine. De plus, dans la perspective des bénéficiaires, les programmes proposés par les institutions financières peuvent aussi démontrer des faiblesses, en matière de conditions d'éligibilité et de critères liés à la bonne santé financière de l'entreprise. De telles conditions peuvent ainsi être interprétées comme des freins importants pour le cédant souhaitant assurer la pérennité de son entreprise par le biais d'une transmission ou pour le repreneur

\section{CONCLUSION}

Cet article a dressé le portrait des programmes d'aide financière en transmission/reprise et a mis en exergue leurs principales forces et faiblesses, permettant ainsi d'avoir une meilleure lecture de l'offre globale existante. D'abord, considérant l'ensemble des programmes retenus dans le cadre de cette étude, nous comprenons que les objectifs convergent en se souciant du maintien de la vitalité du tissu économique régional et national. Malgré leurs spécificités, les programmes existants se démarquent par leur complémentarité, couvrant ainsi l'ensemble de l'offre, tout en distribuant le risque associé à la transmission/reprise des PME. Toujours dans une perspective globale de envisageant de reprendre une entreprise moins saine ou en difficulté.

Enfin, pour les fonds d'investissement, nous avons noté plusieurs forces dont leur expérience, leur réputation, leur portefeuille de financement, leur ouverture à tous types de bénéficiaires et leur complémentarité dans les programmes offerts par l'ensemble des organismes d'aide financière pour la transmission/reprise. Encore une fois, des programmes d'accompagnement, souvent bien rôdés, sont inclus. Par ailleurs, il est important de souligner les difficultés rencontrées par les dirigeants de PME à satisfaire certaines conditions exigées dont le partage du capital-actions et tout ce que cela comporte au chapitre de la gouvernance de l'entreprise. Ralentissement du processus décisionnel; complication de la gestion de l'entreprise; sentiment de perte de liberté, d'autonomie et d'indépendance; crainte de devoir partager de l'information jugée personnelle et confidentielle, obligation de tenir des réunions formelles selon les règles exigées sont les principaux freins exprimés par les propriétaires dirigeants dans la littérature professionnelle et scientifique lorsqu'il est question du partage du capital-actions avec de nouveaux partenaires qui imposent leurs conditions. Encore une fois, nous avons remarqué que l'information sur les programmes est difficilement accessible à tous. Ce manque d'accès peut décourager les éventuels bénéficiaires à frapper à la porte des fonds d'investissement.

l'offre, le financement en transmission/reprise inclut toutes les formes possibles d'aide (certificat de garantie de prêts, prêt conventionnel, participation au capital-actions, congé d'intérêt ou de remboursement pendant une période déterminée, débenture, etc.). L'aide prend aussi la forme d'une gamme de services conseils, de formation ou d'accompagnement, encadrant ainsi le cédant et le repreneur familial, interne ou externe. Enfin, bien que convergente, de l'offre plurielle d'aide financière en transmission/reprise reste imprécise, voire opaque, surtout pour les non initiés. D'autant plus, dans bien des cas, une combinaison de plus d'une source de financement est 
requise. Alors, une meilleure clarté de l'aide offerte aux cédants et aux repreneurs en matière de financement pour les projets de transmission/reprise s'impose. De plus, l'offre d'outils interactifs d'aide au montage financier en transmission/reprise permettant aux éventuels bénéficiaires des décisions les plus éclairées possibles s'avère un défi à relever dans les meilleurs délais.

\section{BIBLIOGRAPHIE}

${ }^{1}$ Cette recherche a été commanditée par le ministère de Développement économique, de l'Innovation et de l'Exportation (MDEIE).

${ }^{2}$ Voir les travaux de Cossette, J. et Mélançon, S. (2010). Le renouvellement de l'entrepreneuriat au Québec: Regard sur 2013 et 2018, Ministère du Développement économique, de l'Innovation et de l'Exportation (MDEIE) et de la Fondation de l'Entrepreneurship. (2010). Indice entrepreneurial québécois 2010: Qu'est-ce que les entrepreneurs québécois ont dans le ventre?

http://www.entrepreneurship.qc.ca/livres-etconferences/livres/indice-entrepreneurial-quebecois2010-0, saisi le 7 février 2011.

${ }^{3}$ Cadieux et Brouard (2009). La transmission des PME : perspectives et enjeux, Québec, Presses de l'Université du Québec.

${ }^{4}$ Voir Lafortune, A., Francoeur, C., Landry, S. (2004). La transmission d'entreprise au Québec : le point de vue des financiers et les produits disponibles, Revue Internationale de Gestion, 29(3), 103-108; Lehmann, P.J. (1993), Le financement de la transmission des PME, Revue Française de Gestion, (95), 116-121; et Senbel, D. et St-Cyr, L. (2007). La transmission d'entreprise: un éclairage sur son financement, Économies et sociétés, K(16), 91-120.

${ }^{5}$ St-Cyr, L., Richer, F., Landry, S., Francoeur, C. (2005). Étude sur l'établissement des meilleures pratiques pour la transmission des entreprises au Québec : aspects financiers et fiscaux, Cahier de recherche 05-02, Chaire de développement et de relève de la PME, HEC Montréal, décembre, 79 pages.

${ }^{6}$ Lors de la rédaction de cet article, Investissement Québec et la Société Générale d'Investissement étaient en processus de fusion.

${ }^{7}$ En 2010, le gouvernement québécois mettait en place le Fonds d'investissement pour la relève agricole (FIRA) en partenariat avec le Mouvement Desjardins et le Fonds de Solidarité. 